\title{
Experimental investigation of photonic crystal waveguide devices and line-defect waveguide bends
}

\author{
M.D.B. Charlton ${ }^{\mathrm{a}, *}$, M.E. Zoorob ${ }^{\mathrm{a}}$, G.J. Parker ${ }^{\mathrm{a}}$, M.C. Netti ${ }^{\mathrm{b}}$, J.J. Baumberg ${ }^{\mathrm{b}}$, \\ S.J. Cox ${ }^{\text {a }}$, H. Kemhadjian ${ }^{\text {a }}$ \\ a Room 2043, Department of Elecectronics and Computer Science, Mountbatten Building, University of Southampton, Highfield, \\ Southampton SO17 1BJ, UK \\ ${ }^{\mathrm{b}}$ Department of Physics and Astronomy, University of Southampton, Highfield, Southampton SO17 1BJ, UK
}

\begin{abstract}
Photonic crystal waveguide devices incorporating line-defect waveguide bends have been fabricated. In this paper we present preliminary experimental analysis of these structures. Although evidence of photonic band-gap effects are observed in the spectra, transmission efficiency was found to be extremely low due to significant up-scattering losses from the holes. In order to quantify this loss mechanism, a detailed experimental and theoretical analysis of scattering effects in regular photonic crystal waveguide devices with band gaps at visible wavelength is presented. Field profiles in line defect structures are analysed using a FDTD (finite difference time domain) method. (C) 2000 Elsevier Science S.A. All rights reserved.
\end{abstract}

Keywords: Photonic crystal; Photonic crystal waveguide; Waveguide splitter

\section{Introduction}

Photonic crystals (PCs) are artificial super-lattice structures, formed by a periodic modulation of dielectric constant which prevent the propagation of photons over a forbidden energy range known as a photonic band gap [1-7].

Important applications for photonic crystals are now emerging in integrated optics. For example the largescale integration of optical devices has so far been inhibited by the problem of guiding light efficiently around tight bends.

It has been predicted that by removing a row of holes from an otherwise regular photonic crystal, a line defect region can be created within the photonic crystal, forming a narrow channel waveguide which may support strongly confined modes within the photonic band gap wavelength range [8-10]. In contrast to conventional waveguides, the method of confinement within the defect waveguide is no longer by a process of total internal reflection, but by a mechanism more analogous

\footnotetext{
* Corresponding author. Tel.: + 44-1703-593737; fax: +44-1703593029.

E-mail address: mdc1@soton.ac.uk (M.D.B. Charlton)
}

to quantum confinement. Due to a lack of available modes within the surrounding porous region, an optical quantum well is formed and light becomes confined to available modes within the defect waveguide region.

Provided that the line defect is sufficiently wide to support a well confined guided mode laterally, modes lying within the band gap wavelength range are expected to follow a sharp bend in the defect waveguide, suffering little (or even zero) loss due to back-reflectance from the corner points $[11,12]$. This facilitates the fabrication of tight waveguide bends for the fabrication of small-scale waveguide splitters and combiners.

Following on from previous experimental work on regular regular photonic crystals etched into silicon Nitride waveguide structures, which were shown to posses a photonic band gap at visible wavelengths, we have fabricated several PBG defect waveguide bend structures based on the same design.

\section{Experimental analysis of photonic crystal lattice}

Before the behaviour of the more complex waveguide bend devices can be understood, it is first important to quantify and understand the characteristics of the regular photonic crystal lattice upon which they are based. 
Several devices consisting of a few rows of holes arranged on a regular triangular lattice of pitch $260 \mathrm{~nm}$ etched through the cladding and core layers (and part into the underlying buffer layer) of a blanket silicon nitride-silicon dioxide waveguide structure were fabricated [13].

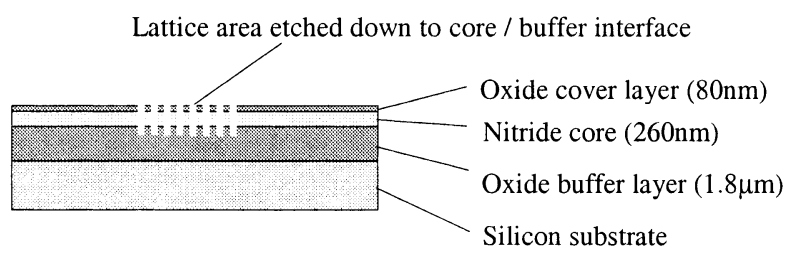

Fig. 1. Cross section of slab waveguide structure.

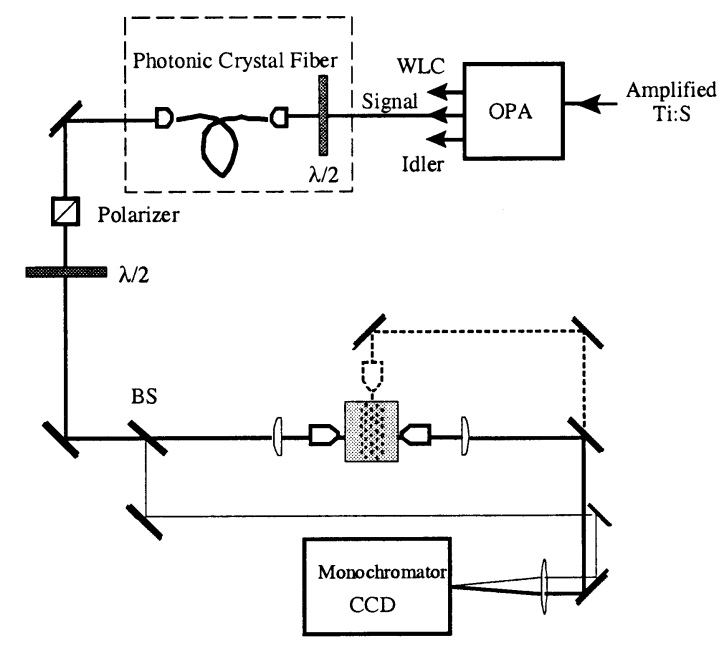

Fig. 2. OPA measurement system. The photonic crystal fiber (dashed box) was used to improve beam stearing and mode stability for the white light continuum only. This was not required when coupling directly fron the OPA.
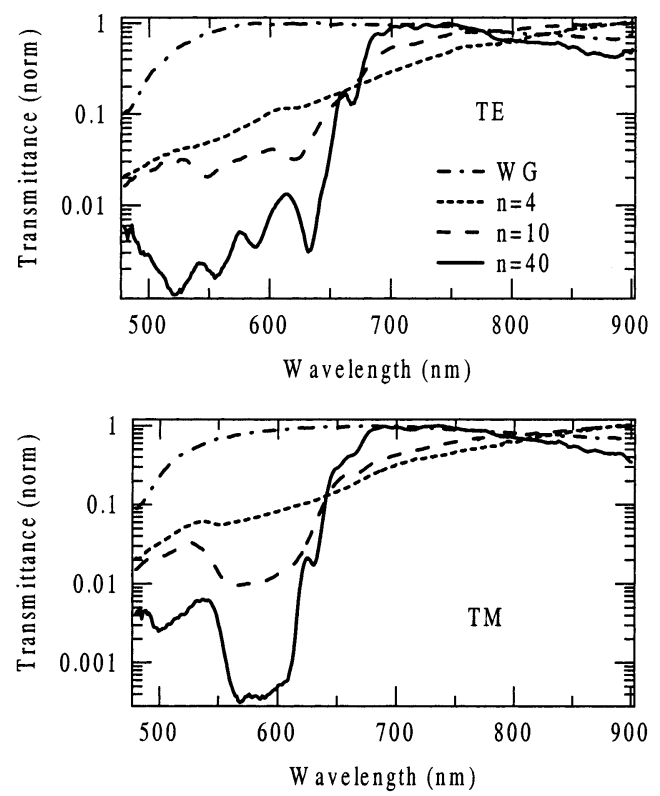

Fig. 3. TE and TM polarised spectra for transmission across a regular photonic crystal lattice with 4,10 and 30 rows of holes.
The basic waveguide structure consists of a $260 \mathrm{~nm}$ thick SiN layer grown on top of a $2 \mu \mathrm{m}$ thick silicon dioxide layer on a silicon substrate. Holes are then patterned by direct write e-beam lithography and plasma etched through the waveguide layers a small distance into the underlying oxide layer (Fig. 1) [13].

Transmission measurements were carried out by spectrally resolving the light propagating through the lattice in different directions. The experiments used a white light continuum produced by focussing $1 \mu \mathrm{J} 100$ fs pulses from a regenerative amplifier tuned to $850 \mathrm{~nm}$ in $1 \mathrm{~mm}$ of sapphire. The availability of such highbrightness ultra-broadband laser sources facilitates high-accuracy transmittance measurements from 450 to $1100 \mathrm{~nm}$ through the waveguide. The use of achromatic optics and a carefully designed photonic-crystal optical fiber spatial filter provided excellent collimation and beam pointing properties for coupling into the planar waveguide. The measurement system is shown schematically in Fig. 2.

Transmission spectra for TE and TM modes propagating across regular devices with 4,10 and 40 rows of holes were measured for several angles of incidence. Spectra for propagation along the $\Gamma-J$ direction are shown in Fig. 3. The spectral response of the supporting slab waveguide structure has been superimposed for reference purposes.

The spectra for the device with four rows of holes shows a classic scattering response for both TE and TM modes across the entire spectrum, and there is little evidence of a band gap opening up. The device with ten rows of holes however clearly shows strong evidence of a photonic band gap for TE modes over the range 540-650 nm (extinction ratio $\sim 10: 1$ ) and for TM modes over the wavelength range $\sim 550-650 \mathrm{~nm}$ (extinction ratio $\sim 12: 1)$. At wavelengths outside the band gap, both spectra follow the classic scattering response (as was the case for four rows of holes), with little additional losses at short wavelengths.

The device with 40 rows of holes however has a significantly different spectra. A band gap is clearly visible over the wavelength range 647-564 $\mathrm{nm}$ for TM modes, with a maximum extinction ratio of $10000: 1$. At wavelengths above the upper band edge, transmission follows the waveguide reference spectra nearly exactly, and there is little evidence of significant scattering loss. At wavelengths below the lower band edge, the spectra again, follows the scattering response curve however, transmission is now significantly weaker than was the case for ten rows of holes. We attribute loss at short wavelengths to increased scattering from the top of the holes. Short wavelength light scattered out of the top of the photonic crystal was visibly found to emerge as a set of discrete beams at fixed angles dependent upon wavelength (this is similar to the behaviour of a 2-di- 
Approximate Band digram for 1st waveguide mode (150 $\mathrm{nm}$ pore diameter $250 \mathrm{~nm}$ core depth $260 \mathrm{~nm}$ pitch)

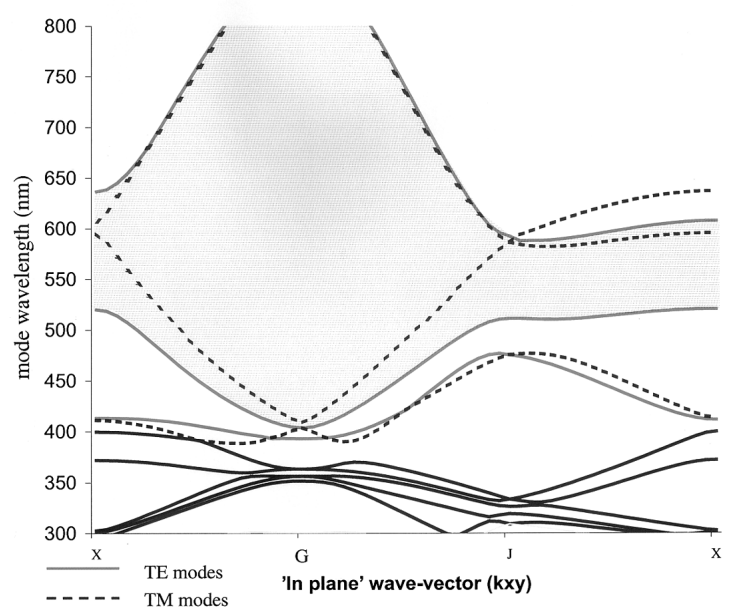

Fig. 4. Approximate band diagram for regular photonic crystal lattice region (3D plane wave analysis assuming metallic boundary conditions).

mensional grating coupler). Light within the photonic band-gap wavelength range was not however scattered out of the top of the structure.

A clear reduction in transmission at $671 \mathrm{~nm}$. Indicates the presence of a band gap for TE modes down to $525 \mathrm{~nm}$. In contrast to the spectra for TM modes, small amplitude dispersive features are observed within the band gap. These are indicative of a TM-TE mode mixing within the band gap. Further analysis of mode mixing and directionality of the band gaps will be published elsewhere.

Scattering loss mechanisms clearly reduce the efficiency of the photonic crystal significantly at short wavelengths. This is likely to present considerable problems for the operation of more complex defect waveguide bends. For this reason, a simplistic analysis of the loss mechanism, and an approximate method of estimation is presented below.

\section{Up scattering loss mechanisms}

A 2-dimensional plane wave model is often used for the analysis of photonic crystals. Briefly: the Helmholtz wave equation is cast as an Eigenvalue problem in reciprocal lattice space. Eigenvalue solutions for a sample $\boldsymbol{k}$-vector then correspond to the free space wavelength of permitted modes. These construct the dispersion curves of the photonic band diagram (Fig. 4). Eigenvector solutions correspond to the amplitude coefficients of constituent Bloch waves $\mathrm{E}$ and $\mathrm{H}$ field profiles can be constructed by inverse Fourier transforming the Bloch expansion over a grid of sample positions in 'real space' across the lattice.
Field plots show that light becomes localised into spatial regions within the lattice, dependent upon lattice geometry, wave polarisation state and wavelength.

Optical energy becomes localised in either the high dielectric regions between the pores or alternatively in the spatial regions of the low dielectric pores themselves. This effect is dependant upon the position of the associated mode with respect to the upper and lower band edges (Fig. 5).

For the case where the field is predominantly localised in the high dielectric regions between the pores, a small proportion of the field energy penetrates across the dielectric pore boundary into the hole. The 2-di-

a)
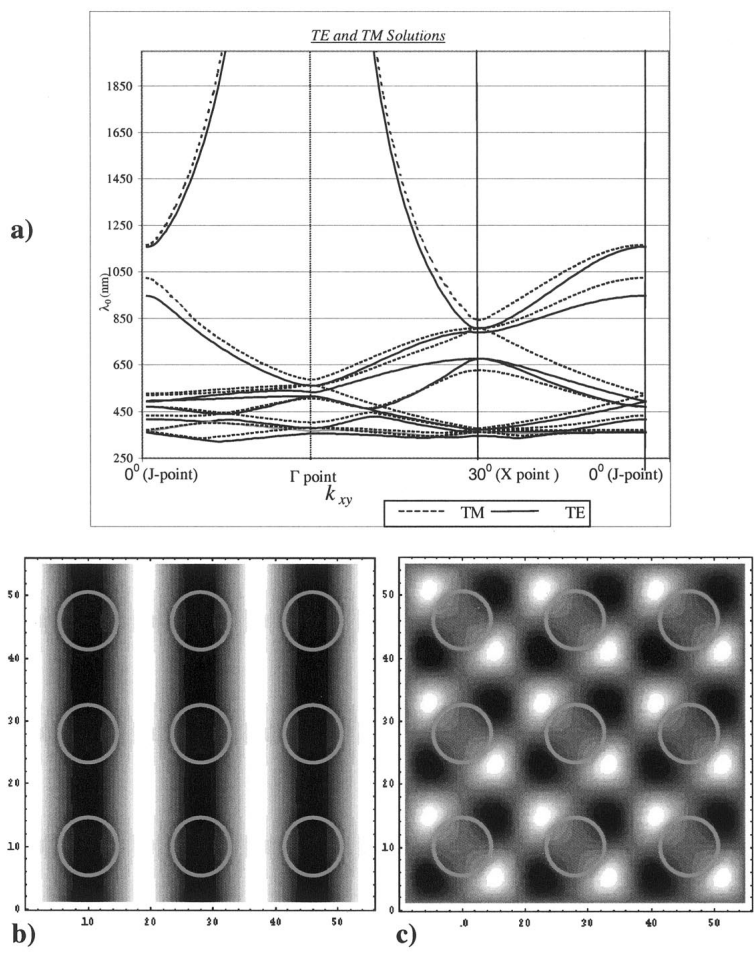

b)

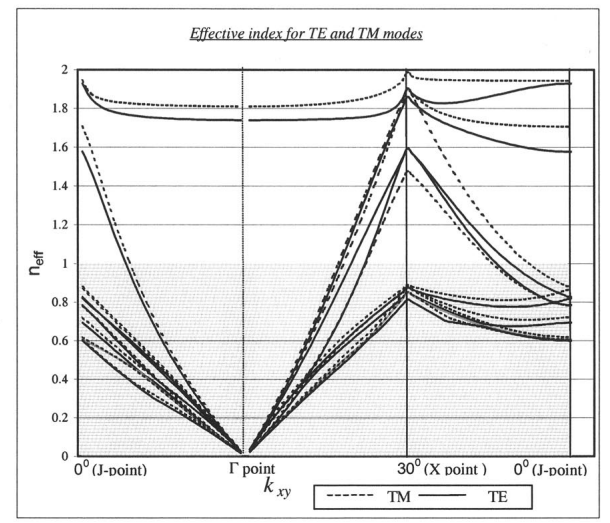

Fig. 5. Field localization effects in a square lattice: (a) band diagram for example structure (2D plane wave analysis); (b) long wave modes on 1st dispersion curve localised in high dielectric regions: these also correspond to high effective index modes (c); (c) shorter wavelenght modes on 3rd dispersion curve are partially localised in the region of the holes; (d) effective mode index of modes supported by example structure. 

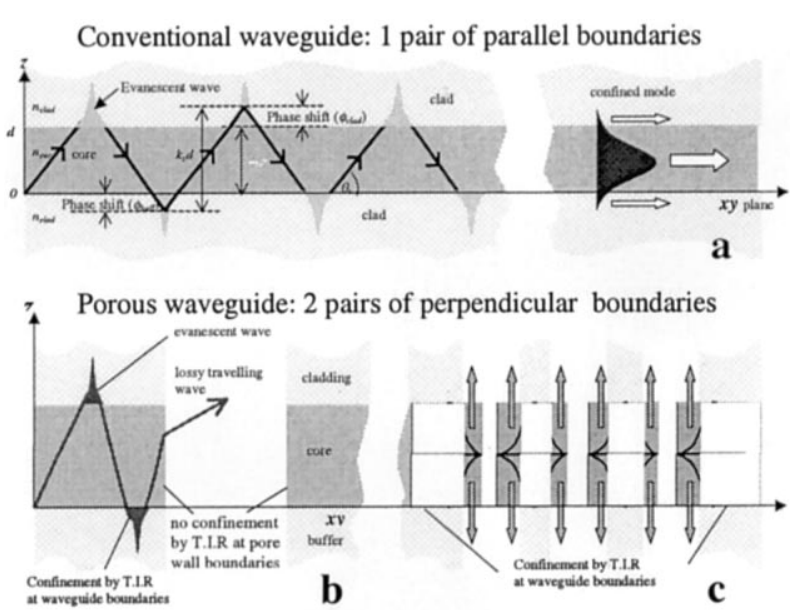

Fig. 6. Waveguide boundary conditions in: (a) standard slab waveguide; (b) porous slab waveguide.

mensiomal plane wave analysis reveals that this forms a stationary wave within the plane of the lattice (Fig. 5b, c).

Now consider wave propagation in a dielectric waveguide. Light is confined by a process of total internal reflection from a pair of dielectric boundaries. In order for this to occur, the mode angle at the waveguide boundaries must be less than the critical angle for total internal reflection (TIR).

If holes are etched through the waveguide core layer, a new pair of perpendicular dielectric boundaries are introduced. It is now impossible for light to be confined at both sets of perpendicular boundaries by TIR (Fig. $6)$.

A wave-function will now be created at the pore wall boundary, which is nominally stationary when resolved within the plane of the lattice (as would be predicted by the 2-dimensional-plane wave simulation Fig. 6c), but which actually has a small propagation vector in the $z$-direction (Fig. 6b).

In effect, mode energy localised in the high dielectric region between the pores will be confined in the $z$-direction by the waveguide boundaries. However, light which the 2-dimensional simulation shows should be localised within the spatial region of the pores will actually leak out of the top and bottom of the pores, due to the absence of a reflective high/low refractive index boundary.

Losses may be reduced by fabricating devices with very small pore diameters. In this case a small portion of the lossy wave may be 'recaptured' at by the opposite pore wall, due to close proximity.

A direct numerical figure for the amount of energy localised in the regions of the holes is given by the value of the effective mode index. This can be obtained from the solutions of either a 2-dimensional (or more accurately 3-dimnesional) plane wave expansion according to the relation: $n_{\text {mode }}=|\boldsymbol{k}| \frac{\lambda_{0}}{2 \pi}$

$n_{\text {mode }}$ is the effective refractive index which the propagating waves experience the porous photonic crystal lattice region to have as they propagate across the photonic crystal region (Fig. 5d).

The maximum and minimum possible values for $n_{\text {mode }}$ are defined by the refractive indexes of the dielectric core material and the holes. In practice the maximum value of $n_{\text {mode }}$ will always be less than that of the high dielectric material, due to the existence of a small field component in the low dielectric regions of the pores. The ratio of reduction in refractive index given by $n_{\text {mode }}$ then gives an approximate measure of the mode energy lost as radiation, (not taking into account the portion of mode energy 're-captured' at the opposite pore wall boundary).

In the long wavelength limit, the effective index of modes lying on the first dispersion curve close to the $\Gamma$ point, tends towards the 'average' value of refractive index [14]. Modes lying on the perimeter of the Brillouin zone are found to have the highest effective mode indexes (incidentally these are modes which exactly satisfy the Bragg condition) and so are expected to be least lossy, and most strongly coupled. This is confirmed experimentally by the spectra shown in Fig. 3 . Transmission for the PC coincides exactly with the reference waveguide spectra at the upper band edge, confirming that coupling is maximised, and scattering losses minimised. Transmission efficiency then reduces gradually as wavelength increases and effective mode index tends towards the 'average' value.

Referring to Fig. 5d, highest effective mode indexes are associated with the first dispersion curve, hence modes lying on the first band are expected to have the lowest loss factor. Modes lying on the second band have significantly lower effective mode indexes, and therefore have a larger field component localised within the regions of the holes, and so are likely to suffer higher losses. Shorter wavelength modes lying on higher order bands have an even larger field component localised within the porous regions, and consequently have much lower effective mode indexes. These effects are shown in Fig. 5a-d.

In a waveguide structure, modes with effective indexes lower than that of the cladding layer will not be confined at all, hence those with $n_{\text {mode }}$ below 1 can be discounted as radiation modes lying outside the waveguides light cone. Those with $n_{\text {mode }}$ slightly above the minimum value of 1 will be scattered strongly out of the waveguide by each row of holes, leading to low transmission for a large number of rows of holes.

In addition to the up-scattering loss mechanism described above, there will also be significant losses associated with impedance missmatch in $n_{\text {mode }}$ across the boundary formed between the porous lattice region and 
the input and output waveguide regions to the sides [13]. Resultant mode miss-match across the boundary leads to radiation loss and partial back-reflections.

All these factors can be taken into account by solving a full 3-dimensional plane wave analysis in combination with the confining waveguide boundary conditions. Values of $n_{\text {mode }}$ thus obtained can be used to obtain an accurate measure of losses due to mode missmatch between the input and output waveguide and the photonic crystal region, using conventional techniques.

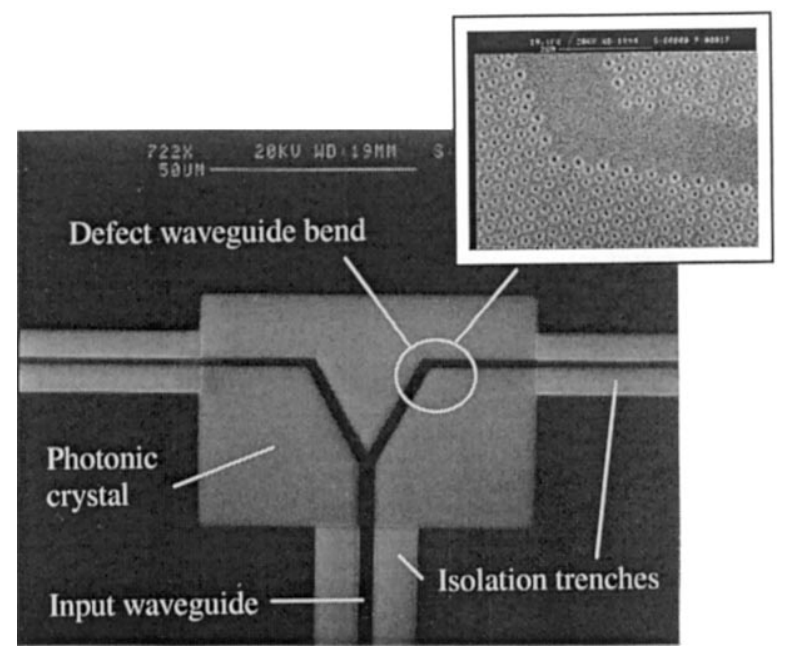

Fig. 7. Example $30-60^{\circ}$ line deefect wavelenght splitter. Example MESA isolated reference structure.

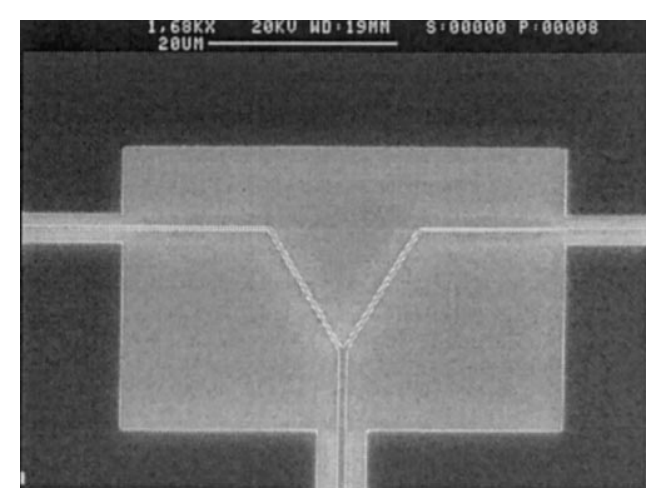

Fig. 8. Example MEASA isolated reference structure.
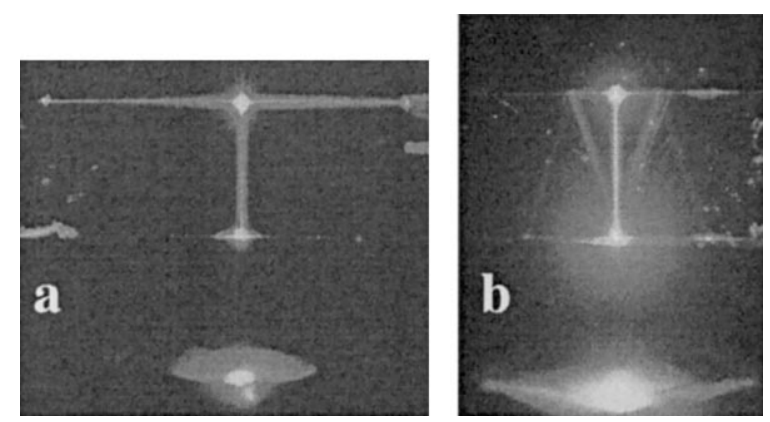

Fig. 9. (a) MESA isolated reference structure transmits significant proportions of light around the bend. (b) PBG defect waveguide device much more lossy.
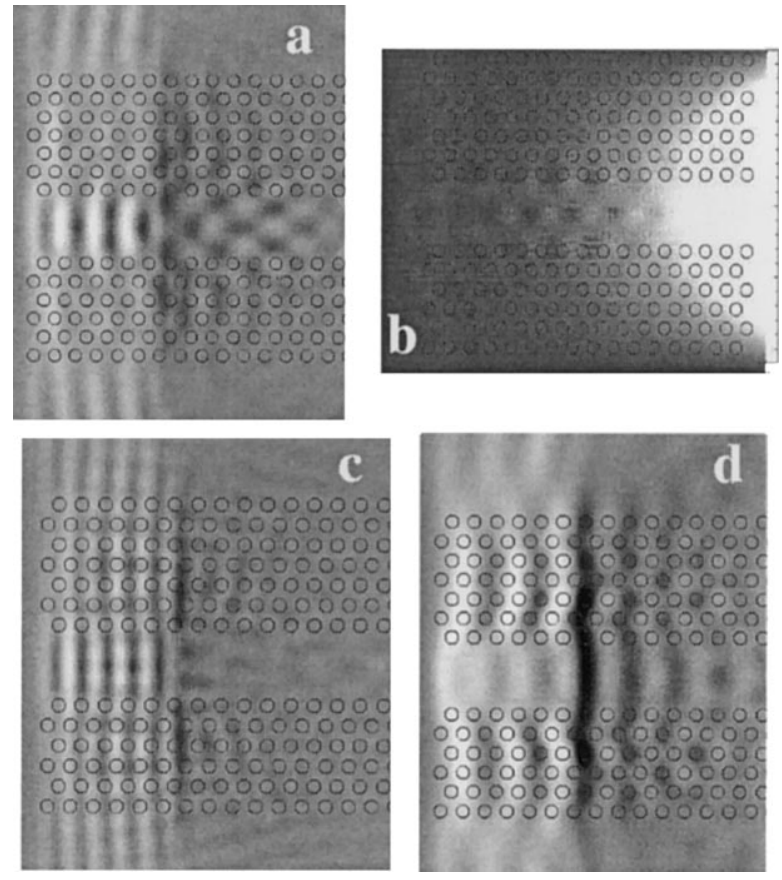

Fig. 10. Snap shot in time of field localisation close to defect waveguide region: (a) $\lambda$-mid-band gap, far from input facet; (b) $\lambda$-mid-band gap at input facet; (c) short $\lambda$, below lower band edge; (d) long $\lambda$, above upper band edge.

\section{Waveguide bends}

Since the spectral characteristics of a $260 \mathrm{~nm}$ pitch triangular lattice of air rods etched into a $250 \mathrm{~nm}$ thick silicon nitride waveguide had been well characterised experimentally, this structure was chosen to form the basis of the first photonic crystal defect waveguide bend devices (Fig. 7).

To facilitate strong optical coupling into the device, a very wide defect waveguide region was incorporated within the lattice by removing 15 rows of holes from the centre of a large rectangular block of photonic crystal. Due to a factor of $\sqrt{3} / 2$ which arises from the triangular lattice geometry, an input waveguide of width $\sim 1.95 \mu \mathrm{m}$, and output waveguides of width $\sim 1.69 \mu \mathrm{m}$ are created. During e-beam lithography, exposure compensation was required for pores positioned close to the defect waveguide region.

To simplify optical coupling into and out of the small device, input and output waveguide channels were defined leading up to the device by etching parallel trenches through the cladding and core layers in the regions to the sides of the photonic crystal lattice (Fig. $10)$.

For reference purposes, MESA isolated waveguide devices with identical geometry were also fabricated (Fig. 8). 
The test devices presented below were slightly different in geometry to the examples shown in Figs. 10 and 11. These had a $90^{\circ}$ bend formed by three $30^{\circ}$ corner junctions. This made the corner as smooth and symmetrical as possible within the limits of the triangular geometry.

Although both structures guided a significant proportion of light around the bend, the PBG devices were found to be extremely lossy in comparison to the MESA isolated reference waveguide structures Fig. 9.

\section{Theoretical modelling}

Two simulation tools are readily available for the analysis of photonic crystal defect structures. The plane wave method can be modified to include a structure factor defining a super-cell, which allows the solution of the dispersion relations for a chosen wave-vector within the defect region [10]. Time averaged field profiles can also be reconstructed using this technique, as described earlier.

Alternatively, a finite difference time domain (FDTD) approach can be used allowing the analysis of wave propagation within the device as a function of time [15]. This approach was used to investigate the distribution of mode energy within a straight defect waveguide section at wavelengths below the lower band edge, above the upper band edge, and at mid gap wavelength (Fig. 10).
Fig. 10b shows the region of the photonic crystal close to the input waveguide facet for a mode lying mid band-gap. For a photonic crystal formed by a lattice of air rods etched into a high dielectric material, a large portion of the mode energy is found to penetrate a large distance into the surrounding photonic crystal lattice.

In effect, the surrounding rows of air rods each act as weak reflectors. This is in contrast to the case described in literature for dielectric rods in air $[11,16]$, where the rods are strongly reflective, maintaining a highly confined mode within the defect waveguide.

Fig. 10a shows field localisation effects some distance away from the input facet at the same mid-gap wavelength. There are two distinct regions to this diagram. On the left-hand side, the forward propagating pulse interferes with light scattered from the photonic crystal region to form standing wave patterns inside the defect waveguide. At this wavelength, mode energy is well confined to the defect waveguide region.

On the right-hand side of the diagram, the forward propagating pulse has already passed, and field patterns formed purely by light scattered back from the photonic crystal lattice are observed. Strong standing wave pattern with artefacts resembling the original lattice shape can be seen. These waves interfere with the forward propagating wave to create the strongly confined standing wave pattern observed to the left.

At this wavelength, several competing modes are maintained laterally within the waveguide due to the
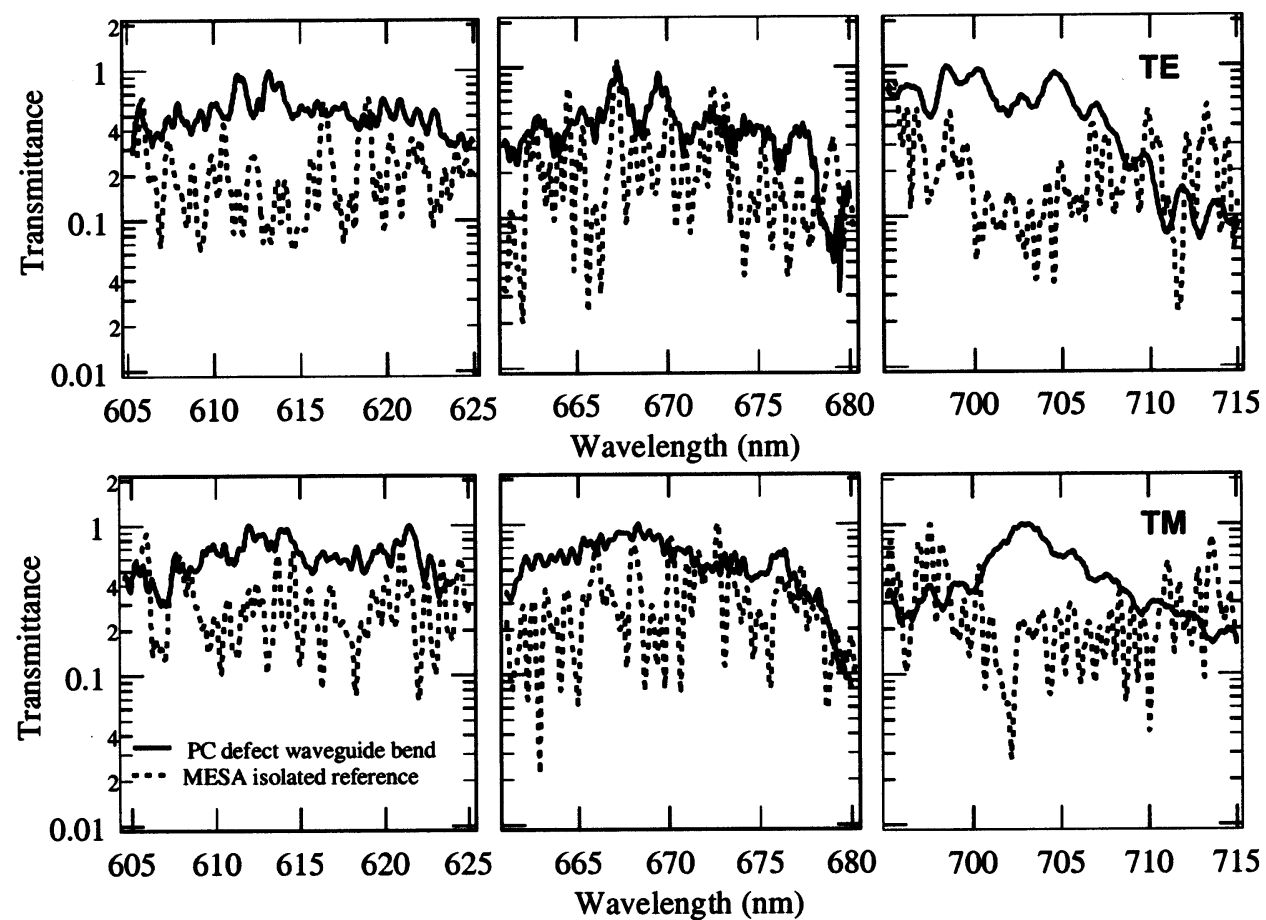

Fig. 11. Comparison between transmission spectra for a photonic crystal waveguide splitter and a MESA isolated waveguide splitter. Spectra were measured using $10 \mathrm{~nm}$ wide OPA pulses. 


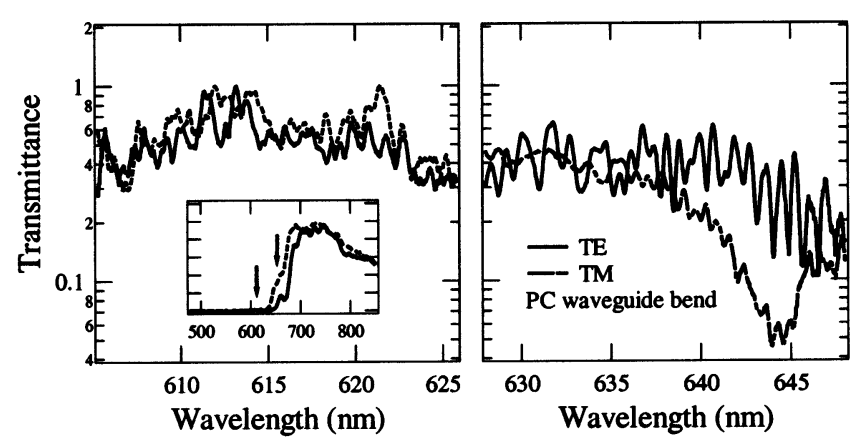

Fig. 12. Comparison between TE and TM transmission spectra for the PC waveguide splitter device. The spectra of the basic photonic crystal lattice is shown as an inset.

large defect waveguide width. Despite the fact that the confined mode lies within the band gap, a small portion of the mode energy still penetrates a significant distance into the PC lattice and will be subject to loss by up-scattering.

At wavelengths far below the lower band edge (Fig. 10c), field energy becomes localised in distinct vertical bands, equally distributed between the defect waveguide region and the surrounding PC lattice. This mode will therefore be subject to much higher scattering loss. At wavelengths far above the upper band edge, (Fig. 10d), mode energy becomes strongly localised in the PC to the sides of the defect waveguide region rather than in the defect waveguide region itself.

\section{Defect waveguide spectra}

Spectral characteristics of the defect waveguide structures were measured by end-fire coupling $10 \mathrm{~nm}$ wide pulses from the tunable OPA laser into the MESA isolated input waveguide (Fig. 2). Spectra were then taken centred about spot wavelengths of $615 \mathrm{~nm}, 670$ $\mathrm{nm}, 705 \mathrm{~nm}$. For these experiments the photonic crystal fiber was not required to improve beam stability.

Fig. 11 shows the transmission spectra for the MESA isolated reference structures (dashed line) super-imposed with the spectra for the PC waveguide splitter (solid line) for TE and TM polarised input modes. Large amplitude high frequency modulation is observed across the entire transmission spectra for the MESA isolated reference structure. The noise was found to be completely independent of input coupling conditions, and is also independent of polarisation state. Due to the large width of the waveguide core, several lateral modes will be supported by the waveguide. The spectral noise can largely be attributed to interference between coupling lateral modes within the waveguide core. In addition, multiple reflections from corner points of the waveguide bend may lead to resonances and more complex mode mixing and interference effects within the waveguide.
The most striking feature of the photonic crystal defect waveguide for both TE and TM modes is the fact that the high frequency spectral noise evident in the reference device spectra has been largely suppressed by the photonic crystal. This is particularly clear for the TM polarised spectra centred at $670 \mathrm{~nm}$, close to the upper band edge of the PBGF (inset to Fig. 12). These results suggest that the $\mathrm{PC}$ defect waveguide has a significantly different internal mode structure to the MESA isolated reference device, and that reflections between the corner points may have been suppressed by the photonic crystal.

Fig. 12 shows a comparison between transmission spectra for TE and TM polarised modes. This time the transmission scale has been expanded to show fine details. For reference purposes the measured transmission spectra for the photonic crystal structure is shown as an inset to show the position of the upper band gap edge in relation to the spectral measurements.

At short wavelengths lying just inside the PBG, there is little variation between the TE and TM spectra. However, as the wavelength traverses the upper band, the amplitude of the spectral noise associated with the reference structure clearly increases, whereas this noise remains well suppressed for TM polarised modes. In fact there is a clear dip in TM transmission at $643 \mathrm{~nm}$. At wavelengths far above the upper band gap, there is little variation in spectral characteristics between $\mathrm{TE}$ and TM modes.

These preliminary spectral results show that there is significant modification of the waveguide spectra due to the presence of the photonic crystal. In addition there is evidence of spectral variations between TE and TM modes close to the upper band gap edge. Current efforts are aimed at probing the structure at wavelengths further inside the band gap.

\section{Conclusions}

A detailed experimental and theoretical analysis of the performance of regular photonic crystal waveguide devices based on a $260 \mathrm{~nm}$ pitch triangular array of air holes etched into a silicon nitride waveguide has been presented.

Photonic crystal devices incorporating line defect waveguides and bends, based on the same design were also fabricated and optically tested.

Spectra for regular photonic crystal show clear evidence of a TM polarised band gap in the wavelength range $550-650 \mathrm{~nm}$, and extinction ratios of 10000:1 were measured. The efficiency of the photonic crystal was also investigated as a function of number of rows of holes. Preliminary experimental results presented in this paper, show that 40 rows of holes is sufficient to give a strong band gap. A more detailed investigation 
into photonic crystal performance as a function of number of rows of holes will be published elsewhere.

It was shown that up-scattering from the holes caused significant losses. This mechanism was described in detail, and a theoretical method for its estimation based on the solution of the effective mode index of guided modes was presented. This was shown to be in good agreement with experimental results for regular lattice structures at long wavelengths.

Photonic crystal line defect waveguide devices based on the same design but incorporating discrete bends was shown for the first time to transmit light around a tight corner over a broad spectral range. However, in comparison with a trench isolated reference waveguide structure with identical geometry the photonic crystal device was found to be extremely lossy.

An FDTD method was used for the investigation of field localisation within the defect-waveguide sections. This showed that the defect waveguide mode had a large field component localised within the surrounding photonic crystal lattice, even at mid-band-gap wavelengths. As was shown to be the case for the regular photonic crystals, up-scattering from the pores is therefore likely to be the major cause of losses in these structures. Quantitative measurements of loss and back-reflectance are currently underway.

Transmission spectra for the defect waveguide devices show unusual polarisation dependent features close to the photonic band gap edge, and a large reduction in spectral noise due to mode mixing.

We suggest that the performance of defect waveguide bends may be improved by utilising a waveguide and photonic crystal geometry which optimises field localisation within the surrounding photonic crystal region to high dielectric regions between the pores, and simul- taneously minimises field penetration into the surrounding photonic crystal lattice at wavelengths of interest.

\section{Acknowledgements}

This work was funded by E.P.S.E.R.C. Thanks to P. Routely for e-beam lithography, M. Josey and T. Blackburn, also Coherent for supplying and setting up the laser system.

\section{References}

[1] E. Yablonovitch, Phys. Rev. Lett. 58 (1987) 2059.

[2] E. Yablonovitch, T.J. Gmitter, Phys. Rev. Lett. 63 (1950) 1989.

[3] E. Yablonovitch, T.J. Gmitter, J. Opt. Soc. Am. A 7 (1990) 1792.

[4] H.W. Lau, G.J. Parker, R. Greef, M. Holling, App. Phys. Lett. 67 (1995) 1877.

[5] M.D.B. Charlton, G.J. Parker, J. Micromech. Microeng. 7 (1997) 155.

[6] U. Gruning, V. Lehmann, App. Phys. Letts 66 (1995) 3254.

[7] U. Gruning, V. Lehmann, S. Ottow, K. Busch, App. Phys. Letts 68 (1995) 747

[8] S.Y. Lin, G. Arjavalingam, J. Opt. Soc. Am. B 11 (1994) 2124.

[9] A.R. McGurn, Phys. Rev. B 53 (1996) 7059.

[10] H. Benisty, J. Appl. Phys. 79 (1996) 7483.

[11] J.D. Joannopoulos, P.R. Villleneuve, S. Fan, Nature 386 (1997) 143.

[12] S. Fan, P.R. Villeneuve, J.D. Joannopoulos, H.A. Haus, Phys. Rev. Lett. 80 (1998) 960.

[13] M.D.B. Charlton, S.W. Roberts, G.J. Parker, Mater. Sci. Eng. B 49 (1997) 155.

[14] M.D.B. Charlton, G.J. Parker, M.E. Zoorob, J. Mater. Sci.: Mater. Electron. 10 (1999) 419.

[15] M.E. Zoorob, M.D.B. Charlton, G.J. Parker, J.J. Baumberg, M.C. Netti, J. Mater. Sci. Eng. B, 1999 (submitted).

[16] J.D. Joannopoulos, R.D. Meade, J.N. Winn, Photonic Crystals, molding the flow of light, Princeton University Press, Princeton, 1995. 